\title{
On continuity properties of semigroups in real interpolation spaces
}

\author{
PeER Christian KunstManNiD
}

Dedicated to Matthias Hieber on the occasion of his 60th birthday.

Abstract. Starting from a bi-continuous semigroup in a Banach space $X$ (which might actually be strongly continuous), we investigate continuity properties of the semigroup that is induced in real interpolation spaces between $X$ and the domain $D(A)$ of the generator. Of particular interest is the case $(X, D(A))_{\theta, \infty}$. We obtain topologies with respect to which the induced semigroup is bi-continuous, among them topologies induced by a variety of norms. We illustrate our results with applications to a nonlinear Schrödinger equation and to the Navier-Stokes equations on $\mathbb{R}^{d}$.

\section{Introduction}

Before I met Matthias Hieber in person (at Karlsruhe in October 1996), I already knew him through his work on integrated semigroups. This concept, which had been propagated by Wolfgang Arendt, comprises strongly continuous semigroups and can be applied to certain operators that do not generate strongly continuous (semi-)groups, e.g., to $i \Delta$ in $L^{p}\left(\mathbb{R}^{d}\right)$ for $p \in[1, \infty] \backslash\{2\}$. Matthias generalized the theory to fractionally integrated semigroups.

A simple example of an operator that generates an $\alpha$-times integrated semigroup for every $\alpha>0$ is the following: Take a Hille-Yosida operator $A$ in a Banach space $X$, i.e., the resolvent set of $A$ contains $(0, \infty)$ and it holds $\sup _{\lambda>0, n \in \mathbb{N}}\left\|\lambda^{n} R(\lambda, A)^{n}\right\|<\infty$, where $R(\lambda, A)=(\lambda-A)^{-1}$ is the resolvent of $A$ at $\lambda$. If the domain $D(A)$ of $A$ is not dense in $X$ then $A$ does not generate a strongly continuous semigroup, but it generates an $\alpha$-times integrated semigroup for every $\alpha>0$ (see [10, Theorem 3.4]). Basic examples for this situation are the translation group or the heat semigroup in $X=L^{\infty}(\mathbb{R})$ or in $X=C_{b}(\mathbb{R})$, the space of bounded and continuous functions $\mathbb{R} \rightarrow \mathbb{C}$. In both cases, the domain of the operator that should be the generator is dense in $B U C(\mathbb{R})$, the space of bounded and uniformly continuous functions $\mathbb{R} \rightarrow \mathbb{C}$, but not in $X$ itself. The semigroups are bounded but lack strong continuity. They are, however, continuous for a weaker topology on $X$. Another concept from the Tübingen

Mathematics Subject Classification: 47 D06, 34 G 10, 35 Q30, 35 Q55

Keywords: Bi-continuous semigroups, Real interpolation, Sectorial operators, Abstract Besov spaces, Nonlinear Schrödinger equations, Navier-Stokes equations. 
school introduced by Kühnemund (see [13]) gives a theoretical framework for these cases: bi-continuous semigroups, i.e., semigroups $(T(t))_{t \geq 0}$ on a Banach space $X$ that are exponentially bounded, but strongly continuous only for a weaker topology $\tau$ on $X$ that still is norming for $X$ and such that norm-closed balls are $\tau$-sequentially complete (we refer to Assumption 2.1 and Definition 2.3 below for the precise formulations, actually we can slightly relax the assumptions on the topology from [13] without changing the results). A natural question then is which topologies $\tau$ can be used for a given semigroup of bounded operators. It seems not to be clear in general if there are any. If there are, there might be many. In this paper we shall see that there is a variety of such topologies in real interpolation spaces between a Banach space $X$ and the domain of the generator of a bi-continuous semigroup, also called abstract Besov spaces. Counterexamples show that the general situation is less favorable. So, somehow semigroups are much better behaved in real interpolation spaces than in the general case.

This is interesting since abstract Besov spaces are better for other properties as well; it is well-known that generators of analytic semigroups have maximal $L^{p}$-regularity (for $1<p<\infty$ ) in real interpolation spaces by a result due to Da Prato-Grisvard, but not in the general case (let me mention that Matthias Hieber was one of the key persons to introduce me to the topic of maximal $L^{p}$-regularity). Remarkable is $\mathrm{G}$. Dore's result that a sectorial operator always has a bounded $H^{\infty}$-functional calculus in the abstract Besov spaces associated with it (see [5] and also [9]), but not in the general case. Our results now add a third one to the list of properties with respect to which operators behave better in abstract Besov spaces associated with it than in the general case.

Since all this may sound very abstract, a word on the motivation for this paper seems to be in order. A topic I met essentially via Matthias (and he was more crucial for this one than for the others mentioned above) is Navier-Stokes equations and Stokes operators. Following Cannone's work on $\mathbb{R}^{d}([3])$ and then Amann's work on domains ([1]) (abstract) Besov spaces became very interesting as spaces for initial values in the theory of Navier-Stokes and similar equations. In particular, Besov spaces with second index $\infty$ play a vital role there. In these spaces, the Stokes operator is not densely defined and the Stokes semigroup is not strongly continuous. Usually, one thus only works on the closure of the domain, where one has a strongly continuous semigroup, or one tries to resort to weak*-continuity of the induced semigroup, which works in some concrete situations but cannot work in general. Here, we want to show that there are other possibilities, and the only available setting, in which such a question can be studied with some satisfaction, is the setting of bi-continuous semigroups.

The paper owes much to our previous work [14] on abstract modulation type spaces where we studied polynomially bounded groups. Nevertheless, there are substantial differences; the functional calculus in [14] is based on the Fourier transform, and we could work with compactly supported $C^{\infty}$-functions on the real line; here, it is based on the Laplace transform, and we have to work with holomorphic functions. Moreover, 
abstract modulation type spaces are instrinsically inhomogeneous, which means that certain difficulties simply cannot arise, whereas here we have both homogeneous and inhomogeneous abstract Besov spaces. A new aspect in the present paper is that, even for a densely defined generator, it may be profitable to consider bi-continuity with respect to a weaker topology. We remark that [2] studied bi-continuity of semigroups that are induced in certain intermediate spaces between a Banach space and the domain of a generator. The approach in [2] to arrive at suitable topologies is different, and the results in [2] and the present paper are disjoint. Finally, it turned out that we had to relax the assumptions on the topology in [13] slightly (see Remarks 2.2 and 2.4). This problem had been overlooked in [14].

Of course, the Stokes semigroup is analytic on a sector. Thus, 0 is the only point where continuity properties become interesting, and some arguments are simpler in this case. Nevertheless, we feel free to study the general situation first and will specify to analytic semigroups later. We shall give some applications of our findings that allow for a greater flexibility when studying nonlinear evolution equations by Banach's contraction principle. Working in a smaller space yields better regularity properties for the fixed point solution, but working in a larger space leads to stronger uniqueness results. So it might be advantageous to have a variety of possibilities.

The paper is organized as follows: In Sect. 2, we gathered the necessary information on bi-continuous semigroups and investigate their Phillips functional calculus. In Sect. 3, we present what we need on extrapolation scales and real interpolation spaces. Section 4 is dedicated to our main result, its discussion and its proof, and Sect. 5 contains applications to Navier-Stokes equations and other problems.

\section{Bi-continuous semigroups}

In this section, we recall basic definitions and properties of bi-continuous (semigroups (we mainly refer to [13] where the theory has its origin). However, we require slightly less on the topology $\tau_{X}$ than had been done in [13].

Assumption 2.1. Let $X$ be Banach space with norm $\|\cdot\|_{X}$ and let $\tau_{X}$ be a locally convex topology on $X$ such that

(i) Any norm-bounded $\tau_{X}$-Cauchy sequence converges in $\left(X, \tau_{X}\right)$.

(ii) The embedding $\left(X,\|\cdot\|_{X}\right) \hookrightarrow\left(X, \tau_{X}\right)$ is continuous.

(iii) Denoting by $\Phi_{X}\left(\tau_{X}\right):=\Phi\left(\tau_{X}\right)$ the set of all linear functionals $\phi$ in the dual space $X^{\prime}:=\left(X,\|\cdot\|_{X}\right)^{\prime}$ with $\|\phi\|_{X^{\prime}} \leq 1$ whose restriction to the closed unit ball $B_{X}$ of $X$ is sequentially $\tau_{X}$-continuous, we have

$$
\|x\|_{X}=\sup _{\phi \in \Phi\left(\tau_{X}\right)}|\langle x, \phi\rangle| \quad \text { for any } x \in X
$$

By (iii), $\tau_{X}$ is necessarily Hausdorff. 
Remark 2.2. Condition (iii) above is slightly weaker than the corresponding condition 3. in [13, Assumption 1] which reads

$$
\|x\|_{X}=\sup \left\{|\langle x, \phi\rangle|: \phi \in\left(X, \tau_{X}\right)^{\prime} \text { and }\|\phi\|_{X^{\prime}} \leq 1\right\} .
$$

Observe that, e.g., for $X=l^{2}(\mathbb{N})$ and the topology of coordinate-wise convergence (i.e., for the restriction of the product topology on $\mathbb{C}^{\mathbb{N}}$ to $\left.l^{2}(\mathbb{N})\right), \phi \in\left(X, \tau_{X}\right)^{\prime}$ if and only if $\phi$ is induced by a finite sequence, whereas the restriction of $\phi$ to $B_{X}$ is $\tau_{X}$-sequentially continuous if and only if $\phi$ is weakly continuous, i.e., induced by a sequence in $l^{2}(\mathbb{N})$. Hence, (iii) is weaker than (2), at least formally. We do not know of a concrete example where (iii) is strictly weaker than (2). The condition (iii) is what is really needed in the proofs of [13], we check this below for the properties we need. We comment on our reasons for the introduction of (iii) in Remark 2.4.

We next recall the definition of a bi-continuous semigroup [13, Def. 3].

Definition 2.3. Suppose that $\tau_{X}$ is as above and that $(T(t))_{t \geq 0}$ is a semigroup with $T(0)=I$ satisfying

$$
\|T(t)\| \leq M e^{\omega t}, \quad t \geq 0,
$$

for some $M \geq 1$ and $\omega \in \mathbb{R}$. Then $(T(t))_{t \geq 0}$ is called bi-continuous (with respect to $\left.\tau_{X}\right)$ if

(i) $[0, \infty) \rightarrow X, t \mapsto T(t) x$ is $\tau_{X}$-continuous for any $x \in X$,

(ii) for any $a>0$ the set $\{T(t): t \in[0, a]\}$ is bi-equicontinuous, i.e., for every $\|\cdot\|$-bounded sequence $\left(x_{n}\right)$ in $X$ that is $\tau_{X}$-convergent to 0 we have

$$
\tau_{X}-\lim _{n \rightarrow \infty} T(t)\left(x_{n}\right)=0
$$

uniformly in $t \in[0, a]$.

Remark 2.4. Our main reason for preferring (1) over (2) is that, for $\phi \in\left(X, \tau_{X}\right)^{\prime}$, the best we can get for $\tilde{\phi}: x \mapsto\langle T(t) x, \phi\rangle$ from Definition 2.3 above is $\tau_{X}$-sequential continuity on norm-bounded subsets and not $\tilde{\phi} \in\left(X, \tau_{X}\right)^{\prime}$. But for $\phi \in \Phi\left(\tau_{X}\right)$ we get $c \tilde{\phi} \in \Phi\left(\tau_{X}\right)$ for some $c>0$. As mentioned above, the proofs in [13] only need (1). Moreover, all other conditions in Assumption 2.1 and Definition 2.3 only need properties of $\tau_{X}$ on norm-bounded sets and only require properties for sequences (see also Remark 2.6), this is not the case for (2) but it is for (1). Finally, the idea behind bi-continuity is the interaction of $\tau_{X}$ with the norm topology and this is somehow not respected by requiring $\phi \in\left(X, \tau_{X}\right)^{\prime}$ in (2).

Following [13, Def. 9], the generator of $(T(t))_{t \geq 0}$ is the unique operator $-A$ on $X$ such that

$$
(\lambda+A)^{-1} x=\int_{0}^{\infty} e^{-\lambda t} T(t) x d t, \quad x \in X, \operatorname{Re} \lambda>\omega .
$$

The integral here is the limit $\lim _{a \rightarrow \infty}$ in operator norm of the integrals $\int_{0}^{a} e^{-\lambda t} T(t) x d t$ which in turn have to be understood as $\tau_{X}$-Riemann integrals (we refer also to [14, 
Prop. A.2] and to the arguments in the proof of Proposition 2.9). Then, (see [13, Cor. 13]) $A$ is bi-closed, i.e., if ( $\left.x_{n}\right)$ is a norm-bounded sequence, $\tau_{X}$-convergent to $x$ and such that $\left(A x_{n}\right)$ is norm-bounded and $\tau_{X}$-convergent to $y$, then $x \in D(A)$ and $A x=y$. Moreover, the domain $D(A)$ of $A$ is bi-dense in $X$, i.e., for any $x \in X$, there is a norm-bounded sequence $\left(x_{n}\right)$ in $D(A)$ that is $\tau_{X}$-convergent to $x$ (see [13, Cor. 13]). We shall check this in Corollary 2.10 as an application of Proposition 2.9.

Examples 2.5. 1. The translation group given by $T(t) f=f(\cdot-t)$ is isometric in $X=L^{\infty}(\mathbb{R})=\left(L^{1}(\mathbb{R})\right)^{\prime}$. It is not strongly continuous, but it is bi-continuous for the weak* topology.

2. More general, if $X$ is not reflexive and $(T(t))_{t \geq 0}=\left(e^{-t A}\right)_{t \geq 0}$ is a $C_{0}$-semigroup in $X$ satisfying (3) then the dual group $\left(T(t)^{\prime}\right)_{t \geq 0}$ in $X^{\prime}$ satisfies (3) as well and is bi-continuous for the weak* topology $\tau_{w^{*}}$ on $X^{\prime}$. Its negative generator is the dual operator $A^{\prime}$ of $A$ whose domain is bi-dense in $X^{\prime}$.

3. The translation group is also not strongly continuous on the space $C_{b}(\mathbb{R})$ of bounded and continuous functions equipped with the sup-norm. But it is bicontinuous for the topology $\tau_{c}$ of uniform convergence on compact subsets of $\mathbb{R}$ (see [13]).

In view of later applications, we want to remark the following with respect to weakor weak*-type topologies.

Remark 2.6. From the definition we see that, for a topology $\tau_{X}$ and a given semigroup $(T(t))_{t \geq 0}$ on $X$, only the restriction of $\tau_{X}$ to norm-closed balls plays a role in Assumption 2.1 and Definition 2.3. In other words, if $\tau_{X}$ and $(T(t))_{t \geq 0}$ satisfy Assumption 2.1 and Definition 2.3 and $\tilde{\tau}_{X}$ is another topology on $X$ such that the restrictions of $\tilde{\tau}_{X}$ and $\tau_{X}$ coincide on norm-closed balls, then also $\tilde{\tau}_{X}$ satisfies Assumption 2.1 and $(T(t))_{t \geq 0}$ is bi-continuous with respect to $\tilde{\tau}_{X}$ as well.

Examples 2.7. 1. The translation semigroup on $L^{\infty}(\mathbb{R})$ is bi-continuous with respect to the topology $\tau_{s}$ of simple convergence in the sense of distributions, i.e., the topology induced by functionals $f \mapsto\langle f, \varphi\rangle$ with $\varphi \in \mathscr{S}(\mathbb{R})$.

2. More general, if $X$ is not reflexive and $(T(t))_{t \geq 0}=\left(e^{-t A}\right)_{t \geq 0}$ is a $C_{0}$-semigroup in $X$ and $D \subseteq X$ is dense in $X$ then the dual group $\left(T(t)^{\prime}\right)_{t \geq 0}$ is bi-continuous for the topology $\tau$ induced by the seminorms $p_{x}\left(x^{\prime}\right):=\left|\left\langle x, x^{\prime}\right\rangle\right|$ with $x \in D$, since $\tau$ restricted to the closed unit ball $B_{X^{\prime}}$ of $X^{\prime}$ coincides with the restriction of $\tau_{w^{*}}$ to $B_{X^{\prime}}$.

3. If $X$ is reflexive, then the weak topology $\tau_{w}$ on $X$ satisfies Assumption 2.1. If $(T(t))_{t \geq 0}$ is a $C_{0}$-semigroup in $X$ then $(T(t))_{t \geq 0}$ satisfies (3) for some $M \geq 1$ and $\omega \in \mathbb{R}$. Moreover, $(T(t))_{t \geq 0}$ is bi-continuous with respect to $\tau_{w}$ : (i) in Definition 2.3 is clear and (ii) holds since

$$
\left\langle T(t) x_{n}, x^{\prime}\right\rangle=\left\langle x_{n}, T(t)^{\prime} x^{\prime}\right\rangle
$$

and $\left\{T(t)^{\prime} x^{\prime}: t \in[0, a]\right\}$ is compact in $X^{\prime}$ by strong continuity of $\left(T(t)^{\prime}\right)_{t \geq 0}$ in $X^{\prime}$. As in 2. above we may replace $\tau_{w}$ by a topology $\tau$ induced by $p_{x^{\prime}}(x):=$ $\left|\left\langle x, x^{\prime}\right\rangle\right|$ where $x^{\prime} \in D$ and $D \subseteq X^{\prime}$ is dense. 
The example in 3. may seem artificial for the moment, but we shall see that it increases the applicability of our main result Theorem 4.1. We refer to Remark 4.3.

Throughout the paper, we assume the following.

Assumption 2.8. Let $\left(X,\|\cdot\|_{X}\right)$ be a Banach space and $\tau_{X}$ a topology on $X$ satisfying Assumption 2.1. We assume that $(T(t))_{t \geq 0}$ is a semigroup in $X$ that satisfies (3) for $\omega=0$ and is bi-continuous with respect to the topology $\tau_{X}$. We denote the generator of $(T(t))_{t \geq 0}$ by $-A$ and write $T(t)=e^{-t A}$.

The following proposition on the Phillips functional calculus for the semigroup $(T(t))_{t \geq 0}$ is a variant of [14, Prop. 4.4]. We remark that [12, Theorem 2.2] states the Phillips functional calculus for bi-continuous semigroups but without the assertion on bi-equicontinuity below, which is crucial for our purposes.

We denote by $\mathscr{M}$ the set of all complex Borel measures $\mu$ on $[0, \infty)$ and by $\mathscr{L} \mathscr{M}$ the set of their Laplace transforms. We recall that, for $\mu \in \mathscr{M}$ its Laplace transform $\mathscr{L} \mu$ is given by $\mathscr{L} \mu(\lambda):=\int_{[0, \infty)} e^{-\lambda t} d \mu(t)$ for $\lambda \in \mathbb{C}$ with $\operatorname{Re} \lambda>0$. We shall use that for a $\tau_{X}$-continuous and norm-bounded function $f:[a, b] \rightarrow X$, where $0 \leq a<b$, and a complex Borel measure $\mu \in \mathscr{M}$, the integral $\int_{[a, b]} f(t) d \mu(t)$ exists in a $\tau_{X}$-Riemann sense: The $\tau_{X}$-limit of sums

$$
\sum_{j=1}^{n} f\left(\xi_{j}\right) \mu\left(\left[t_{j-1}, t_{j}\right)\right)+f(b) \mu(\{b\}),
$$

where $a=t_{0}<t_{1}<\ldots<t_{n}=b$ and $\xi_{j} \in\left[t_{j-1}, t_{j}\right)$, exists as $\max _{j}\left|t_{j}-t_{j-1}\right|$ tends to 0 . For a proof of this fact, we refer to [14, Proposition A.2]. Moreover, also $\int_{(a, b]} f(t) d \mu(t)$ exists in such a sense as $\tau_{X}$-limit of sums

$$
f\left(\xi_{1}\right) \mu\left(\left(a, t_{1}\right)\right)+\sum_{j=2}^{n} f\left(\xi_{j}\right) \mu\left(\left[t_{j-1}, t_{j}\right)\right)+f(b) \mu(\{b\}),
$$

as $\max _{j}\left|t_{j}-t_{j-1}\right|$ tends to 0 . It is clear that for $0 \leq a<c<b$, we then have

$$
\int_{[a, b]} f(t) d \mu(t)=\int_{[a, c]} f(t) d \mu(t)+\int_{(c, b]} f(t) d \mu(t) .
$$

Proposition 2.9. (Phillips calculus) For $\mu \in \mathscr{M}(\mathbb{R})$ and $x \in X$ the limit

$$
(\mathscr{L} \mu)(A) x:=\lim _{a \rightarrow \infty} \int_{[0, a]} T(t) x d \mu(t)
$$

exists in operator norm and $x \mapsto(\mathscr{L} \mu)(A) x$ defines a linear operator $(\mathscr{L} \mu)(B) \in$ $L(X)$ satisfying

$$
\|(\mathscr{L} \mu)(A)\|_{L(X)} \leq M|\mu|([0, \infty)) .
$$

The map $\mathscr{L} \mathscr{M} \rightarrow L(X), \mathscr{L} \mu \mapsto(\mathscr{L} \mu)(A)$ is an algebra homomorphism. Moreover, if $\mathcal{F} \subseteq \mathscr{M}$ is a subset bounded in variation norm and satisfies

$$
\lim _{a \rightarrow \infty} \sup _{\mu \in \mathcal{F}}|\mu|((a, \infty))=0
$$

then $\{(\mathscr{L} \mu)(A): \mu \in \mathcal{F}\}$ is bi-equicontinuous. 
Proof. Let $\mu \in \mathscr{M}$ and $x \in X$. For $a>0$, the integral $\int_{[0, a]} T(t) x d \mu(t)$ exists in a $\tau_{X}$-Riemann sense. By Assumption 2.1 (iii), we have

$$
\left\|\int_{[0, a]} T(t) x d \mu(t)\right\|_{X}=\sup _{\phi \in \Phi\left(\tau_{X}\right)}\left|\int_{[0, a]}\langle T(t) x, \phi\rangle d \mu(t)\right| \leq M|\mu|([0, a])\|x\|_{X} .
$$

Similarly, we have for $0<a<b$,

$$
\left\|\int_{(a, b]} T(t) x d \mu(t)\right\|_{X}=\sup _{\phi \in \Phi\left(\tau_{X}\right)}\left|\int_{(a, b]}\langle T(t) x, \phi\rangle d \mu(t)\right| \leq M|\mu|((a, b])\|x\|_{X} .
$$

Denoting the operator $x \mapsto \int_{[0, a]} T(t) x d \mu(t)$ by $\int_{[0, a]} T(t) d \mu(t)$ and defining $\int_{(a, b]} T(t) d \mu(t)$ similarly these estimates imply $\int_{[0, a]} T(t) d \mu(t) \in L(X)$ and that

$$
\lim _{a \rightarrow \infty} \int_{[0, a]} T(t) d \mu(t), \quad \lim _{b \rightarrow \infty} \int_{(a, b]} T(t) d \mu(t)
$$

exist in operator norm. Denoting the first limit by $(\mathscr{L} \mu)(A)$ as in the assertion, the estimates show (5). Denoting the second limit by $\int_{(a, \infty)} T(t) d \mu(t)$ we clearly have

$$
\begin{aligned}
& (\mathscr{L} \mu)(A)=\int_{[0, a]} T(t) d \mu(t)+\int_{(a, \infty)} T(t) d \mu(t), \quad\left\|\int_{(a, \infty)} T(t) d \mu(t)\right\|_{X} \\
& \leq M|\mu|((a, \infty))
\end{aligned}
$$

For the following we observe that for $\phi \in \Phi\left(\tau_{X}\right)$, we have

$$
\begin{aligned}
& \langle(\mathscr{L} \mu)(A) x, \phi\rangle=\lim _{a \rightarrow \infty}\left\langle\int_{[0, a]} T(t) x d \mu(t), \phi\right\rangle=\lim _{a \rightarrow \infty} \int_{[0, a]}\langle T(t) x, \phi\rangle d \mu(t) \\
& =\int_{[0, \infty)}\langle T(t) x, \phi\rangle d \mu(t),
\end{aligned}
$$

where the last integral is a Lebesgue integral.

Since linearity is clear, it remains to show multiplicativity for the algebra property, i.e., for $\mu, v \in \mathscr{M}$, we have to show $(\mathscr{L} \mu)(A)(\mathscr{L} v)(A)=(\mathscr{L}(\mu * v))(A)$. We do this by applying functionals $\phi \in \Phi\left(\tau_{X}\right)$ :

$$
\begin{aligned}
\langle(\mathscr{L}(\mu * v))(A) x, \phi\rangle & =\int_{[0, \infty)}\langle T(t) x, \phi\rangle d(\mu * v)(t) \\
& =\int_{[0, \infty)} \int_{[0, \infty)}\langle T(t+s) x, \phi\rangle d \mu(t) d v(s) \\
& =\int_{[0, \infty)}\left\langle T(s) \int_{[0, \infty)} T(t) x d \mu(t), \phi\right\rangle d v(s) \\
& =\langle(\mathscr{L} v)(A)(\mathscr{L} \mu)(A) x, \phi\rangle .
\end{aligned}
$$

Now suppose that $\mathcal{F} \subseteq \mathscr{M}$ is bounded in variation norm and satisfies (6). Let $\left(x_{n}\right)$ be a norm-bounded sequence that is $\tau_{X}$-convergent to 0 . We may assume $\left\|x_{n}\right\| \leq 1$. Let 
$p$ be a continuous seminorm and $\varepsilon>0$. We may assume $p \leq\|\cdot\|_{X}$. By assumption we find $a>0$ such that

$$
\sup _{\mu \in \mathcal{F}}|\mu|((a, \infty)) \leq \varepsilon .
$$

For $\mu \in \mathcal{F}$ we write

$$
p\left((\mathscr{L} \mu)(A) x_{n}\right) \leq p\left(\int_{[0, a]} T(t) x_{n} d \mu(t)\right)+\left\|\left(\int_{(a, \infty)} T(t) d \mu(t)\right) x_{n}\right\|_{X} .
$$

Using $\tau_{X}$-continuity of $p$ and (7), we obtain

$$
\begin{aligned}
p\left((\mathscr{L} \mu)(A) x_{n}\right) & \leq \int_{[0, a]} p\left(T(t) x_{n}\right) d|\mu|(t)+M|\mu|((a, \infty)) \\
& \leq \sup _{t \in[0, a]} p\left(T(t) x_{n}\right) \sup _{\mu \in \mathcal{F}}|\mu|([0, \infty))+M \varepsilon .
\end{aligned}
$$

By Assumption 2.8, we have $\sup _{t \in[0, a]} p\left(T(t) x_{n}\right) \rightarrow 0$ as $n \rightarrow \infty$, and this implies the assertion since $\varepsilon>0$ was arbitrary.

Taking $\mu_{z}=e^{-t z} d t$ where $\operatorname{Re} z>0$ we have $\mathscr{L} \mu_{z}(\lambda)=(z+\lambda)^{-1}$ and $\mathscr{L} \mu_{z}(A)=$ $(z+A)^{-1}$ (as we shall check immediately below). Taking $\mu=\delta_{t}$ where $t>0$, we have $\mathscr{L} \delta_{t}(\lambda)=e^{-\lambda t}$ and $\mathscr{L} \delta_{t}(A)=T(t)$.

Corollary 2.10. For $\operatorname{Re} z>0$ let $R_{z}:=\mathscr{L} \mu_{z}(A)$ where $\mu_{z}:=e^{-t z} d t$. Then $\left(R_{z}\right)_{\operatorname{Re} z>0}$ is the resolvent of a closed linear operator $-A$ in $X$ that is bi-closed and whose domain is bi-dense.

Proof. By Proposition 2.9, $\left(R_{z}\right)_{\operatorname{Re} z>0}$ is a pseudo-resolvent, since $\mu_{z} * \mu_{w}=(w-$ $z)\left(\mu_{z}-\mu_{w}\right)$ for $\operatorname{Re} z, \operatorname{Re} w>0$. For $x \in X, z>0, \delta>0$, and a $\tau_{X}$-continuous seminorm $p \leq\|\cdot\|_{X}$, we have

$$
\begin{aligned}
p\left(z R_{z} x-x\right) & \leq \int_{0}^{\delta} z e^{-z t} p(T(t) x-x) d t+(M+1) \int_{\delta}^{\infty} z e^{-z t} d t \\
& \leq \sup _{t \in[0, \delta]} p(T(t) x-x)+(M+1) \int_{\delta z} e^{-t} d t .
\end{aligned}
$$

Hence, $p\left(z R_{z} x-x\right) \rightarrow 0$ as $z \rightarrow \infty$. We conclude that each $R_{z}$ is injective, and thus $R_{z}=(z+A)^{-1}$ for some closed operator $A$ in $X$. Bi-density of $D(A)$ follows from $\tau_{X}-\lim _{z \rightarrow \infty} z(z+A)^{-1} x=x$. Finally, if $\left(x_{n}\right)$ is a bounded sequence in $D(A)$ such that $\left(A x_{n}\right)$ is bounded and $x_{n} \rightarrow x, A x_{n} \rightarrow y$ with respect to $\tau_{X}$ then Proposition 2.9 yields $(1+A)^{-1} x_{n} \rightarrow(1+A)^{-1} x, A(1+A)^{-1} x_{n}=(1+A)^{-1} A x_{n} \rightarrow(1+A)^{-1} y$ with respect to $\tau_{X}$. However, we also have $A(1+A)^{-1} x_{n}=x_{n}-(1+A)^{-1} x_{n} \rightarrow$ $x-(1+A)^{-1} x$ with respect to $\tau_{X}$. Hence $x-(1+A)^{-1} x=(1+A)^{-1} y$, and we conclude $x \in D(A)$ and $A x=y$. 


\section{Extrapolation scales and real interpolation spaces}

\subsection{Extrapolation scales}

We assume that $A$ is a sectorial operator in $X$ with domain $D(A)$ and range $R(A)$. We also assume that $A$ is injective. In contrast to the case of reflexive spaces (see [4, Thm. 3.8] or [11, p. 857]), if $A$ is not injective in $X$, we can only resort to $\varepsilon+A$ for some $\varepsilon>0$. The following construction of extrapolation spaces is well-established (see, e.g., [9, Sect. 6.3]). For $k \in \mathbb{N}$ we equip $D\left(A^{k}\right)$ with the norm $\|x\|_{X_{k}(A)}:=\left\|(1+A)^{k} x\right\|_{X}$. Then $\left(X_{k}(A),\|\cdot\|_{X_{k}(A)}\right)$ is Banach space and the restriction of $1+A$ to $X_{k}(A)$ defines an isometry $X_{k}(A) \rightarrow X_{k-1}(A)$ if we let $X_{0}(A):=\left(X,\|\cdot\|_{X}\right)$. We extend this scale to negative integers by first extending $1+A:\left(D(A),\|\cdot\|_{X}\right) \rightarrow\left(X,\left\|(1+A)^{-1} \cdot\right\|_{X}\right)$ to an isometry $\left(X,\|\cdot\|_{X}\right) \rightarrow\left(X_{-1}(A),\|\cdot\|_{X_{-1}(A)}\right)$. We thus have a version of $A$ in $X_{-1}(A)$ that has the same properties in $X_{-1}(A)$ that $A$ has in $X$, and is an extension of the original operator $A$. We can proceed in the same way to construct $X_{-2}(A)$ from $X_{-1}(A)$ etc, and obtain a scale of Banach spaces $X_{k}(A), k \in \mathbb{Z}$.

Since $A$ is in addition injective, $\|x\|_{\dot{X}_{1}(A)}:=\|A x\|_{X}$ defines a norm on $D(A)$, and we can extend $A:\left(D(A),\|\cdot\|_{\dot{X}_{1}(A)}\right) \rightarrow\left(X,\|\cdot\|_{X}\right)$ to an isometry $\left(\dot{X}_{1}(A), \| \cdot\right.$ $\left.\|_{\dot{X}_{1}(A)}\right) \rightarrow\left(X,\|\cdot\|_{X}\right)$. Similarly as before, we can also extend $A:\left(D(A),\|\cdot\|_{X}\right) \rightarrow$ $\left(X,\left\|A^{-1} \cdot\right\|_{X}\right)$ to an isometry $\left(X,\|\cdot\|_{X}\right) \rightarrow\left(\dot{X}_{-1}(A),\|\cdot\|_{\dot{X}_{-1}(A)}\right)$. By iteration, we obtain a scale of Banach spaces $\dot{X}_{k}(A)$ for $k \in \mathbb{Z}$ (with $\dot{X}_{0}(A)=\left(X,\|\cdot\|_{X}\right)$ ) such that suitable versions of $A$ act as isometries $\dot{X}_{k}(A) \rightarrow \dot{X}_{k-1}(A)$. Observe also that, e.g., $\dot{X}_{-1}(A)=\dot{X}_{1}\left(A^{-1}\right)$ and so on. We remark that $X_{-1}(A)=X+\dot{X}_{-1}(A)$, $X_{1}(A)=X \cap \dot{X}_{1}(A)$ etc, and that one can complete the picture (cf. the diagram below) to a lattice of vector spaces in the set theoretic sense. Within this lattice we identify another scale of spaces letting, for $k \in \mathbb{N}, \widehat{X}_{k}(A):=\dot{X}_{k}(A) \cap \dot{X}_{-k}(A)$ and $\widehat{X}_{-k}(A):=\dot{X}_{k}(A)+\dot{X}_{-k}(A)$ such that a suitable version of $A(1+A)^{-2}$ acts as an isometry $\widehat{A}_{k-1}(A) \rightarrow \widehat{X}_{k}(A)$ for all $k \in \mathbb{Z}$. Then, for any space $Y$ in the lattice, there is $k \in \mathbb{N}$ such that $\widehat{X}_{k}(A) \hookrightarrow Y \hookrightarrow \widehat{X}_{-k}(A)$. Hence, $\widehat{X}_{-\infty}(A):=\bigcap_{k \in \mathbb{N}} \widehat{X}_{-k}(A)$ is an ambient space for the lattice, and any space contains $\widehat{X}_{\infty}(A):=\bigcap_{k \in \mathbb{N}} \widehat{X}_{k}(A)$. The following diagram shows some of these spaces.

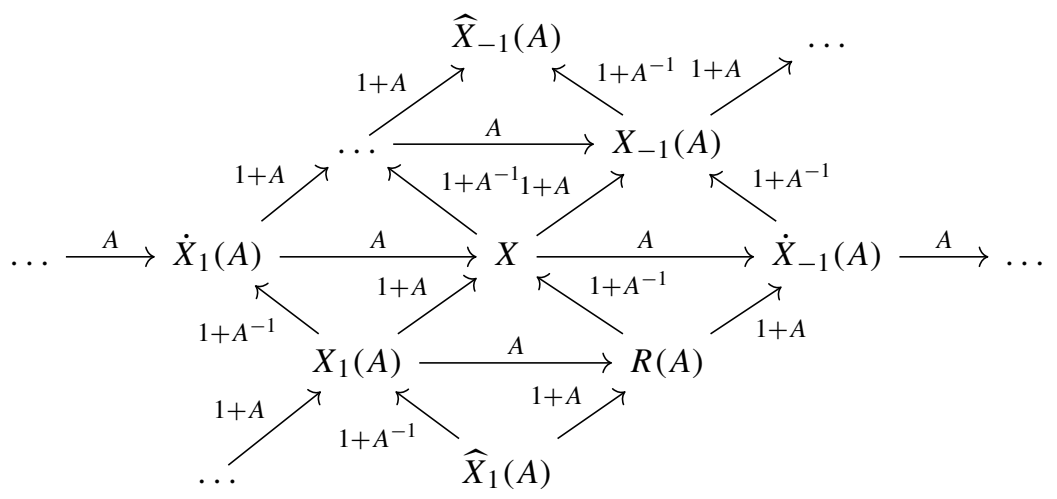


For more details on the construction, we refer to [9, Sect. 6.3] and [8] (in [9] the space $\widehat{X}_{-\infty}(A)$ is denoted $\left.U\right)$. We remark that in case $0 \in \rho(A)$, we have $\widehat{X}_{k}(A)=$ $X_{k}(A)=\dot{X}_{k}(A)$ for all $k \in \mathbb{Z}$, and the lattice degenerates to a linear chain.

In our situation, $A$ is the negative generator of a bounded semigroup $(T(t))_{t \geq 0}$ which is bi-continuous with respect to the topology $\tau_{X}$. By the isometries in the lattice, this semigroup induces a bounded semigroup in each of the spaces $Y$ in the lattice, which is bi-continuous with respect to $\tau_{Y}$, where $\tau_{Y}$ is the topology $\tau$ transferred to $Y$ by the corresponding isometry $X \rightarrow Y$. All the semigroup operators are consistent, and via Proposition 2.9 also their Phillips functional calculi are consistent.

\subsection{Real interpolation spaces}

We are interested in real interpolation spaces between two spaces $\dot{X}_{k}(A)$ and $\dot{X}_{l}(A)$ in the homogeneous scale of abstract Besov spaces associated with $A$. We refer to [ 9 , Section 6.4] for the background. Recall that $A$ is the negative generator of a bicontinuous semigroup in $X$ with respect to the topology $\tau_{X}$, and we assume that $A$ is injective. Hence, $A$ is sectorial of angle $\frac{\pi}{2}$ and injective. We fix the function

$$
\psi(\lambda):=c_{0} e^{-\left(\lambda^{1 / 2}+\lambda^{-1 / 2}\right)}, \quad \lambda \in \mathbb{C} \backslash((-\infty, 0]),
$$

where $c_{0}>0$ is such that $\int_{0}^{\infty} \psi(t) \frac{d t}{t}=1$. Observe that $\lambda \mapsto \lambda^{k} \psi(\lambda)$ is bounded on every sector $\Sigma_{\theta}, \theta \in(0, \pi)$. One can thus define bounded operators $\psi(t A), t>0$, by the holomorphic functional calculus for sectorial operators, and they are bounded $X \rightarrow \widehat{X}_{k}(A)$ for any $k \in \mathbb{N}$. As a consequence they map $\widehat{X}_{-\infty}(A) \rightarrow \widehat{X}_{\infty}(A)$. Now we show that the operators $\psi(t A)$ are in the Phillips calculus of Proposition 2.9. ${ }^{1}$

Lemma 3.1. There exists $f \in L^{1}\left(\mathbb{R}_{+}\right)$such that $\mathscr{L} f=\psi$. Hence, for each $t>0$, there exists $f_{t} \in L^{1}\left(\mathbb{R}_{+}\right)$with $\mathscr{L} f_{t}(\lambda)=\psi(t \lambda)$. More general, for any $k, l, m \in \mathbb{Z}$ and $t>0$, there exists a function $g_{k, l, m, t} \in L^{1}\left(\mathbb{R}_{+}\right)$such that $\mathscr{L}_{k, l, m, t}(\lambda)=$ $\lambda^{k}(1+\lambda)^{l}\left(1+\lambda^{-1}\right)^{m} \psi(t \lambda)$.

Proof. We clearly have $\sup _{s>0}\|\psi(s+i \cdot)\|_{L^{2}}<\infty$. By Paley-Wiener, the inverse Fourier transform of $\xi \mapsto \psi(i \xi)$ is a function $f \in L^{2}(\mathbb{R})$ with supp $f \subseteq[0, \infty)$. Since $\xi \mapsto \frac{d}{d \xi} \psi(i \xi)$ is in $L^{2}(\mathbb{R})$, we conclude $f \in L^{1}(\mathbb{R})$ and $\mathscr{L} f=\psi$. Then, we put $f_{t}:=t^{-1} f(\cdot / t)$. The final statement is proved in the same way.

Remark 3.2. By [9, Theorem 5.2.6], we have

$$
\begin{aligned}
\int_{a}^{b} \psi(t A) x \frac{d t}{t} & =\left(\int_{a}^{b} \psi(t \lambda) \frac{d t}{t}\right)(A) x \rightarrow \int_{0}^{\infty} \psi(t A) x \frac{d t}{t} \\
& =x \quad(a \rightarrow 0+, b \rightarrow \infty)
\end{aligned}
$$

for all $x \in \overline{D(A) \cap R(A)}$ (convergence in the norm of $X$ ). Recalling our lattice of extrapolation spaces, we conclude that we have convergence for any $x \in X$ in the

\footnotetext{
${ }^{1}$ In [11, Lemma 1] the authors dealt with a similar problem.
} 
norm of $\widehat{X}_{-1}(A)$ and more general: if $x \in \widehat{X}_{-\infty}(A)$ belongs to a space $Y$ in the lattice, then we have convergence in the norm of $\widehat{Y}_{-1}(A):=(1+A)\left(1+A^{-1}\right) Y$ (the first space vertically above $Y$ ).

We shall work with the following description of the real interpolation space between homogeneous abstract Besov space associated with $A$. The statement follows from the results in [9, Section 6.4].

Proposition 3.3. Let $k, l \in \mathbb{Z}$ such that $l<k$. Let $\theta \in(0,1)$ and $p \in[1, \infty]$. Then $\left(\dot{X}_{l}(A), \dot{X}_{k}(A)\right)_{\theta, p}=\left\{x \in \widehat{X}_{-\infty}(A): t \mapsto t^{-\theta(k-l)} \psi(t A) x \in L^{p}\left(\mathbb{R}_{+}, d t / t, \dot{X}_{l}(A)\right)\right\}$ and

$$
\begin{aligned}
x & \mapsto\left\|t \mapsto t^{-\theta(k-l)} \psi(t A) x\right\|_{L^{p}\left(\mathbb{R}_{+}, d t / t, \dot{X}_{l}(A)\right)} \\
& =\left\|t \mapsto t^{-\theta(k-l)} A^{l} \psi(t A) x\right\|_{L^{p}\left(\mathbb{R}_{+}, d t / t, X\right)}
\end{aligned}
$$

defines an equivalent norm on $\left(\dot{X}_{l}(A), \dot{X}_{k}(A)\right)_{\theta, p}$.

\section{Main result}

Recall that $\tau_{X}$ satisfies Assumption 2.1 on the Banach space $X$ and that $A$ is the negative generator of a bi-continuous semigroup $(T(t))_{t \geq 0}$ on $X$ with respect to the topology $\tau_{X}$ and $A$ is injective. The following is our main result.

Theorem 4.1. Let $k, l \in \mathbb{Z}$ such that $l<k, m \in \mathbb{N}, \theta \in(0,1)$ and $p \in[1, \infty]$. Assume that $Y$ is a Banach space satisfying

$$
\left(\dot{X}_{l}(A), \dot{X}_{k}(A)\right)_{\theta, p} \hookrightarrow Y \hookrightarrow \widehat{X}_{-m}(A) .
$$

Assume that the semigroup operators $(T(t))_{t \geq 0}$, considered as operators on $\widehat{X}_{-m}(A)$, restricted to $Y$ induce a bi-continuous semigroup on $Y$ for a topology $\tau_{Y}$ such that the embedding

$$
\left(B_{Y},\left.\tau_{Y}\right|_{B_{Y}}\right) \hookrightarrow\left(\widehat{X}_{-m}(A), \tau_{\widehat{X}_{-m}(A)}\right)
$$

is continuous, where $B_{Y}$ denotes the closed unit ball of $Y$. Then, the semigroup $(T(t))_{t \geq 0}$ on $\left(\dot{X}_{l}(A), \dot{X}_{k}(A)\right)_{\theta, p}$ is bi-continuous for the restriction of the topology $\tau_{Y}$ to $\left(\dot{X}_{l}(A), \dot{X}_{k}(A)\right)_{\theta, p}$.

We remark that (10) is weaker than continuity of the embedding $\left(Y, \tau_{Y}\right)$ $\hookrightarrow\left(\widehat{X}_{-m}(A), \tau_{\widehat{X}_{-m}(A)}\right)$ (see Remark 2.6). Observe that by (9), we have $B_{Y} \subseteq C B_{\widehat{X}_{-m}(A)}$ for some $C>0$. Before we give the proof of Theorem 4.1, we want to illustrate the result.

Remark 4.2. Assume $0 \in \rho(A)$ and $l=m=0, p=\infty$. Then, $k \in \mathbb{N}$.

1. We can take $\left(Y, \tau_{Y}\right)=\left(X, \tau_{X}\right)$ and, for any $\theta \in(0,1), k \in \mathbb{N}$, the semigroup $(T(t))_{t \geq 0}$ is bi-continuous on $\left(X, X_{k}(A)\right)_{\theta, \infty}$ for the restriction of the topology $\tau_{X}$ to $\left(X, X_{k}(A)\right)_{\theta, \infty}$. 
2. We actually have $\left(X, X_{k}(A)\right)_{\theta, p} \hookrightarrow \overline{D(A)}$ (closure in the norm of $X$ ), and on $\overline{D(A)}$ the semigroup $(T(t))_{t \geq 0}$ is strongly continuous. So we can take the norm topology of $X$ and restrict it to $\left(X, X_{k}(A)\right)_{\theta, \infty}$, and obtain that $(T(t))_{t \geq 0}$ is bi-continuous on $\left(X, X_{k}(A)\right)_{\theta, \infty}$ with respect to $\|\cdot\|_{X}$, which may come as a surprise.

3. We can also take $Y:=\left(X, X_{k}(A)\right)_{\sigma, q}$ where $\sigma \in(0, \theta)$ and $q \in[1, \infty)$. Then, the semigroup $(T(t))_{t \geq 0}$ is strongly continuous in $Y$, and we obtain that $(T(t))_{t \geq 0}$ is bi-continuous on $\left(X, X_{k}(A)\right)_{\theta, \infty}$ with respect to $\|\cdot\|_{\left(X, X_{k}(A)\right)_{\sigma, q}}$, another surprise. We can include the case $q=\infty$ in the last statement, since the semigroup $(T(t))_{t \geq 0}$ is strongly continuous on the closure $Y$ of $\left(X, X_{k}(A)\right)_{\theta, \infty}$ in $\left(X, X_{k}(A)\right)_{\sigma, \infty}$.

Remark 4.3. In Theorem 4.1, we assume that $A$ is the negative generator of a $C_{0^{-}}$ semigroup and that $\tau_{X}$ is the norm topology on $X$. Then, $\tau_{\widehat{X}_{-m}(A)}$ is the norm topology on $\widehat{X}_{-m}(A)$, and condition (10) seems to be a bit restrictive. However, recalling Remark 2.6 and Example 2.7.3, we may use weaker topologies $\tau_{X}$ than the norm topology on $X$ in order to allow for more topologies $\tau_{Y}$ on $Y$. This is the reason behind the formulation of condition (10) above, and it is also the motivation to include the case $p \in[1, \infty)$ in Theorem 4.1, namely to allow for weaker topologies also in spaces where $(T(t))_{t \geq 0}$ acts as a $C_{0}$-semigroup.

We give some concrete examples, starting with a counterexample.

Example 4.4. Consider a scaled version of right translation $(T(t) f)(x):=e^{-t} f(x-$ $t$ ) on $X=C_{b}(\mathbb{R})$ which is bi-continuous for the topology $\tau_{c}$ of uniform convergence on compact subsets of $\mathbb{R}$ (see Example 2.5.1). The negative generator is $A=\frac{d}{d x}+1$ with $D(A)=\left\{f \in C^{1}(\mathbb{R}) \cap C_{b}(\mathbb{R}): f^{\prime} \in C_{b}(\mathbb{R})\right\}$, notice that $0 \in \rho(A)$. Let $Y$ be the closure of $D(A)$ in $C_{b}(\mathbb{R})$ and $Z:=D(A)$ with the graph norm. Then, $\left(\left.T(t)\right|_{Y}\right)_{t \geq 0}$ is a $C_{0}$-semigroup in $Y$, i.e. bi-continuous with respect to $\|\cdot\|_{\infty}$. However, the topology $\tau_{Z}$ induced by $\|\cdot\|_{\infty}$ on $Z$ does not satisfy Assumption 2.1 (i): Let $f(x):=|x|$ for $|x| \leq 1$ and extend $f$ to a 2-periodic function $f: \mathbb{R} \rightarrow \mathbb{C}$. The function $f$ has a weak derivative $f^{\prime} \in L^{\infty}(\mathbb{R})$ with $\left\|f^{\prime}\right\|_{\infty}=1$. Take $\rho \in C^{\infty}$ with $\rho \geq 0$ and support $\subseteq[-1,1]$ and $\int \rho d x=1$, let $\rho_{n}:=n \rho(n \cdot)$ and $f_{n}:=\rho_{n} * f$. Then $\left\|f_{n}\right\|_{\infty}=1$, $\left\|f_{n}^{\prime}\right\|_{\infty} \leq 1$ and $\left\|f_{n}-f\right\|_{\infty} \rightarrow 0$. Hence, $\left(f_{n}\right)$ is bounded in $Z$ and $\left(f_{n}\right)$ is $\tau_{Z}$-Cauchy, but the $\|\cdot\|_{\infty}$-limit $f$ of the sequence $\left(f_{n}\right)$ does not belong to $Z$.

The next example shows that Theorem 4.1 also includes cases of weak* continuous semigroups in real interpolation spaces with index $\infty$.

Example 4.5. Consider $A=1-\Delta$ in $X=L^{q}\left(\mathbb{R}^{d}\right)$ where $q \in(1, \infty)$. Then, $0 \in \rho(A),-A$ is the generator of a scaled version of the heat semigroup, and $\dot{X}_{k}(A)=X_{k}(A)=H^{2 k, q}\left(\mathbb{R}^{d}\right)$ are Bessel potential spaces for $k \in \mathbb{Z}$. Moreover, the real interpolation spaces (with $\theta \in(0,1)$ )

$$
\left(X, \dot{X}_{k}(A)\right)_{\theta, \infty}=B_{q, \infty}^{2 k \theta}\left(\mathbb{R}^{d}\right)
$$


are Besov spaces. It is well-known that for the usual $L^{2}$-duality, one has $B_{q, \infty}^{2 k \theta}\left(\mathbb{R}^{d}\right)=$ $\left(B_{q^{\prime}, 1}^{-2 k \theta}\left(\mathbb{R}^{d}\right)\right)^{\prime}$ and that $\mathscr{S}\left(\mathbb{R}^{d}\right)$ is dense in $B_{q^{\prime}, 1}^{-2 k \theta}\left(\mathbb{R}^{d}\right)$ (we refer to [16]). By Remark 2.6, simple convergence in the sense of distributions is as good as the weak* topology $\tau_{w^{*}}$ on $B_{q, \infty}^{2 k \theta}\left(\mathbb{R}^{d}\right)$, and we see that (10) holds for $m=0$ and $k \in \mathbb{N}$ if we take for $\tau_{X}$ the weak topology on $X$ (which is as good as simple convergence in the sense of distributions, too).

Example 4.6. In the situation of Example 4.5, let $k \in \mathbb{N}$. Then, $\left(e^{-t A}\right)_{t \geq 0}$ is a bicontinuous semigroup on $B_{q, \infty}^{2 k \theta}\left(\mathbb{R}^{d}\right)$ for the norm of $L^{q}$ and for the norm of $B_{q, p}^{2 k \sigma}\left(\mathbb{R}^{d}\right)$ if $\sigma \in(0, \theta)$ and $p \in[1, \infty]$.

Proof of Theorem 4.1. Let $\left(x_{n}\right)$ be a sequence in $Z:=\left(\dot{X}_{l}(A), \dot{X}_{k}(A)\right)_{\theta, p}$. We equip $Z$ with the norm (8) from Proposition 3.3, which we call $\|\cdot\|_{Z}$, and assume $\left\|x_{n}\right\|_{Z} \leq M$ and that $\left(x_{n}\right)$ is $\tau_{Y}$-Cauchy. By (10), $\left(x_{n}\right)$ is $\tau_{\widehat{X}_{-m(A)}}$-Cauchy in $\widehat{X}_{-m}(A)$, hence $\tau_{\widehat{X}_{-m}(A)^{-}}$ convergent to some $x \in \widehat{X}_{-m}(A)$. By Proposition 2.9 and Lemma 3.1, we then have that for any $t>0,\left(A^{l} \psi(t A) x_{n}\right)$ is $\tau_{X}$-convergent to $A^{l} \psi(t A) x$. For $\phi \in \Phi\left(\tau_{X}\right)$, we thus have

$$
\begin{aligned}
& \left|\left\langle t^{-\theta(k-l)} A^{l} \psi(t A) x, \phi\right\rangle\right|=\lim _{n \rightarrow \infty}\left|\left\langle t^{-\theta(k-l)} A^{l} \psi(t A) x_{n}, \phi\right\rangle\right| \\
& \quad \leq \liminf _{n \rightarrow \infty}\left\|t^{-\theta(k-l)} A^{l} \psi(t A) x_{n}\right\|_{X} .
\end{aligned}
$$

Using Assumption 2.1 (iii) we infer, for each $t>0$,

$$
\left\|t^{-\theta(k-l)} A^{l} \psi(t A) x\right\|_{X} \leq \liminf _{n \rightarrow \infty}\left\|t^{-\theta(k-l)} A^{l} \psi(t A) x\right\|_{X},
$$

which (by Fatou's Lemma in case $p<\infty$ ) leads to $\|x\|_{Z} \leq M$. Hence, $x \in Z$, and Assumption 2.1 (i) is verified for $\left.\tau_{Y}\right|_{Z}$ in $Z$. Assumption 2.1 (ii) holds for $\left.\tau_{Y}\right|_{Z}$ in $Z$ since (9) and Assumption 2.1 (ii) for $\tau_{Y}$ in $Y$ imply

$$
\left(Z,\|\cdot\|_{Z}\right) \hookrightarrow\left(Y,\|\cdot\|_{Y}\right) \hookrightarrow\left(Y, \tau_{Y}\right) .
$$

We verify Assumption 2.1 (iii) for $\left.\tau_{\widehat{X}_{-m}(A)}\right|_{Z}$ in $Z$ which implies (iii) for $\left.\tau_{Y}\right|_{Z}$ in $Z$ by (10). We start with the case $p<\infty$ and denote by $D$ the set of step functions $g$ of the form

$$
g=\sum_{j=1}^{n} \beta_{j} \phi_{j} 1_{\left[a_{j-1}, a_{j}\right)},
$$

where $n \in \mathbb{N}, \phi_{j} \in \Phi\left(\tau_{X}\right), \beta_{j} \in \mathbb{C}, 0<a_{0}<a_{1}<\ldots<a_{n}<\infty$, and $\|g\|_{L^{p^{\prime}}\left(d t / t ; X^{\prime}\right)} \leq 1$. It is not hard to see that for any $f \in L^{p}(d t / t ; X)$,

$$
\|f\|_{L^{p}(d t / t ; X)}=\sup _{g \in D}\left|\int_{0}^{\infty}\langle f(t), g(t)\rangle \frac{d t}{t}\right|
$$

(check this on step functions and use (iii) for $\tau_{X}$ in $X$ ). A function $g \in D$ induces a linear functional $\phi_{g}$ by

$$
\left\langle x, \phi_{g}\right\rangle=\int_{0}^{\infty}\left\langle t^{-\theta(k-l)} A^{l} \psi(t A) x, g(t)\right\rangle \frac{d t}{t},
$$


which is actually defined on $\widehat{X}_{-\infty}(A)$, since the support of $g$ is contained in an interval $[a, b] \subseteq(0, \infty)$. We clearly have $\left\|\phi_{g}\right\|_{Z^{\prime}} \leq 1$. Now sequential $\tau_{\widehat{X}_{-m}(A)}$-continuity of $x \mapsto\left\langle x, \phi_{g}\right\rangle$ on $B_{\widehat{X}_{-m}(A)}$ is equivalent to sequential $\tau_{X}$-continuity of

$$
\begin{aligned}
x & \mapsto\left\langle(1+A)^{m}\left(1+A^{-1}\right)^{m} x, \phi_{g}\right\rangle \\
& =\int_{a}^{b}\left\langle t^{-\theta(k-l)} A^{l}(1+A)^{m}\left(1+A^{-1}\right)^{m} \psi(t A) x, g(t)\right\rangle \frac{d t}{t},
\end{aligned}
$$

on $B_{X}$, which follows from Proposition 2.9 and Lemma 3.1. By (11), we thus obtain

$$
\begin{aligned}
& \sup _{g \in D}\left|\left\langle x, \phi_{g}\right\rangle\right|=\sup _{g \in D}\left|\int_{0}^{\infty}\left\langle t^{-\theta(k-l)} A^{l} \psi(t A) x, g(t)\right\rangle \frac{d t}{t}\right| \\
& \quad=\left\|t \mapsto t^{-\theta(l-k)} A^{l} \psi(t A) x\right\|_{L^{p}(d t / t ; X)}=\|x\|_{Z} .
\end{aligned}
$$

We have verified Assumption 2.1 for $\tau_{\widehat{X}_{-m}(A)} \mid Z$ in $Z$ in case $p<\infty$.

In case $p=\infty$, we denote by $D$ the set of functions $g$ of the form $(b-a)^{-1} \phi 1_{[a, b]}$ where $\phi \in \Phi\left(\tau_{X}\right)$ and $0<a<b<\infty$. Then, we have (11) for $L^{\infty}(d t / t ; X)$, at least for functions $f$ that are $\|\cdot\|_{X}$-continuous, which is the case relevant for us. We define functionals $\phi_{g}$ for $g \in D$ as in (12) and can proceed as before to finish the verification of Assumption 2.1 (iii).

It is clear that $\left(\left.T(t)\right|_{Z}\right)_{t \geq 0}$ is a bounded semigroup in $Z$ by interpolation. Properties (i) and (ii) in Definition 2.3 for $\left.\tau_{Y}\right|_{Z}$ in $Z$ follow easily from the corresponding properties for $\tau_{Y}$ in $Y$ and $Z \subseteq Y$.

\section{Applications}

We give two applications to nonlinear problems. We shall use the following facts: If $\tau_{X}$ is a topology on a Banach space $X$ that satisfies Assumption 2.1 and $a>0$ then

$$
C_{\tau_{X}}([0, a], X):=\left\{f:[0, a] \rightarrow X: f \text { is }\|\cdot\|_{X} \text {-bounded and } \tau_{X} \text {-continuous }\right\}
$$

is a Banach space for the norm $\|f\|_{\infty}:=\sup _{t \in[0, a]}\|f(t)\|_{X}$, i.e., a closed subspace of the Banach space of all $\|\cdot\|_{X}$-bounded functions (this is similar to [6, Lemma 3.2]). If, in addition, $(T(t))_{t \geq 0}$ is a bi-continuous semigroup on $X$ with respect to $\tau_{X}$ then

$$
T(\cdot) *: C_{\tau_{X}}([0, a], X) \rightarrow C_{\tau_{X}}([0, a], X), \quad f \mapsto\left[t \mapsto \int_{0}^{t} T(t-s) f(s) d s\right]
$$

is continuous with

$$
\left\|\int_{0}^{t} T(t-s) f(s) d s\right\|_{X} \leq M t \sup _{s \in[0, t]}\|f(s)\|_{X}, \quad t \in[0, a] .
$$

This relies on $\tau_{X}$-continuity of $t \mapsto T(t-s) f(s)$ on $[0, t]$ and the usual norm estimate. 


\subsection{A nonlinear Schrödinger equation in Besov spaces}

We consider the cubic nonlinear Schrödinger equation on $\mathbb{R}$

$$
\left\{\begin{aligned}
i \partial_{t} u+\Delta u & = \pm|u|^{2} u, \quad t>0 \\
u(0) & =u_{0}
\end{aligned}\right.
$$

Here, $A=-i \Delta$ and $-A$ is the generator of the unitary group $(T(t))_{t \in \mathbb{R}}:=\left(e^{i t \Delta}\right)_{t \in \mathbb{R}}$ in $X=L^{2}(\mathbb{R})$. We have $X_{1}(A)=H^{2,2}(\mathbb{R})$ and $\left(X, X_{1}(A)\right)_{\theta, \infty}=B_{2, \infty}^{2 \theta}(\mathbb{R})$ for $\theta \in(0,1)$. For $\alpha>\frac{1}{2}, Z:=B_{2, \infty}^{\alpha}(\mathbb{R})$ embeds into $C_{0}(\mathbb{R})$ and is a Banach algebra. Hence, $u \mapsto|u|^{2} u=u \bar{u} u$ is continuous on $Z$ and $\left\||u|^{2} u\right\|_{Z} \leq C\|u\|_{Z}^{3}$. We look for mild solutions of (13), i.e., for solutions $u(\cdot)$ of the fixed point equation

$$
u(t)=T(t) u_{0} \mp i \int_{0}^{t} T(t-s)\left(|u(s)|^{2} u(s)\right) d s, \quad t \in[0, a] .
$$

By Theorem 4.1, $(T(t))_{t \geq 0}$ is bi-continuous on $Z$ with respect to the following topologies $\tau_{Z}$ : the weak* topology induced by $B_{2,1}^{-\alpha}(\mathbb{R}) ;$ the norm topology of $L^{2}(\mathbb{R}) ;$ the norm topology of $B_{2, p}^{\beta}(\mathbb{R})$ for $\beta \in(0, \alpha)$ and $p \in[1, \infty]$ (notice that $B_{2,2}^{\beta}(\mathbb{R})=$ $\left.H^{\beta, 2}(\mathbb{R})\right)$.

Proposition 5.1. Let $u_{0} \in B_{2, \infty}^{\alpha}(\mathbb{R})=: Z$ where $\alpha>\frac{1}{2}$ und let $\tau_{Z}$ be the norm topology of $B_{2, p}^{\beta}(\mathbb{R})$ where $\beta \in\left(\frac{1}{2}, \alpha\right)$ and $p \in[1, \infty]$. Then, there exists $a>0$ such that (14) has a solution, which is unique in a closed ball in $C_{\tau_{Z}}([0, a], Z)$.

Proof. We use Banach's fixed point theorem and only have to check that $u \mapsto|u|^{2} u$ is sequentially $\tau_{Z}$-continuous on $\|\cdot\|_{Z}$-bounded sets. This holds since $B_{2, p}^{\beta}(\mathbb{R})$ is a Banach algebra by $\beta>\frac{1}{2}$.

5.2. Mild solutions of Navier-Stokes equations in Besov spaces

Let $d \in \mathbb{N}$ with $n \geq 3$. We consider the Navier-Stokes equations on $\mathbb{R}^{d}$.

$$
\left\{\begin{aligned}
\partial_{t} u-\Delta u+(u \cdot \nabla) u+\nabla \pi & =0 & & \text { in }(0, T) \times \mathbb{R}^{d}, \\
\operatorname{div} u & =0 & & \text { in }(0, T) \times \mathbb{R}^{d}, \\
u(0, \cdot) & =u_{0} & & \text { on } \mathbb{R}^{d} .
\end{aligned}\right.
$$

We apply the Helmholtz projection $\mathbb{P}$ to (15) and obtain the following abstract Cauchy problem

$$
\left\{\begin{aligned}
\dot{u}(t)+A u(t) & =-\mathbb{P} \operatorname{div}(u(t) \otimes u(t)), \quad t>0, \\
u(0) & =u_{0},
\end{aligned}\right.
$$

where $A=-\mathbb{P} \Delta$ denotes the Stokes operator on a function space of divergence-free vector fields. Recall that on $\mathbb{R}^{d}$, the Laplacian $\Delta$ commutes with $\mathbb{P}$. Again, we look 
for mild solutions, i.e., solutions of the fixed point problem

$$
u(t)=T(t) u_{0}-\int_{0}^{t} T(t-s) \mathbb{P}(\operatorname{div}(u(s) \otimes u(s))) d s, \quad t \in[0, a] .
$$

where $(T(t))_{t \geq 0}=\left(e^{-t A}\right)_{t \geq 0}$ denotes the Stokes semigroup, which, on $\mathbb{R}^{d}$, is a restriction of the heat semigroup $\left(e^{t \Delta}\right)_{t \geq 0}$.

Proposition 5.2. Let $q \in(d, \infty)$. If the initial value

$$
u_{0} \in B_{q, \infty, \sigma}^{-1+d / q}\left(\mathbb{R}^{d}\right):=\left\{f \in B_{q, \infty}^{-1+d / q}\left(\mathbb{R}^{d}\right): \operatorname{div} f=0\right\}
$$

has sufficiently small norm then there exists a $>0$ such that (17) has a unique solution in

$$
C_{\tau}\left([0, a], B_{q, \infty, \sigma}^{-1+d / q}\left(\mathbb{R}^{d}\right) \cap L_{1 / 2-d /(2 q)}^{\infty}\left(0, a ; L_{\sigma}^{q}\left(\mathbb{R}^{d}\right)\right),\right.
$$

where $\tau$ denotes one of the following topologies on $B_{q, \infty, \sigma}^{-1+d / q}\left(\mathbb{R}^{d}\right)$ : the weak ${ }^{*}$-topology induced by functionals $\langle\cdot, g\rangle$ with $g \in B_{q^{\prime}, 1, \sigma}^{1-d / q}\left(\mathbb{R}^{d}\right)$; the topology of simple convergence in the sense of distributions induced by functionals $\langle\cdot, \varphi\rangle$ with divergence-free $\varphi \in$ $C_{c}^{\infty}\left(\mathbb{R}^{d}\right)^{d}$; the norm topology of $B_{q, \infty}^{-\beta}\left(\mathbb{R}^{d}\right)^{d}$ with $\beta \in(1-d / q, 1)$; the norm topology of $H^{-1, q}\left(\mathbb{R}^{d}\right)^{d}$.

Proof. We recall that $B_{q, \infty}^{-1+d / q}\left(\mathbb{R}^{d}\right)^{d}=\left(H^{-2, q}\left(\mathbb{R}^{d}\right)^{d}, L^{q}\left(\mathbb{R}^{d}\right)^{d}\right)_{\frac{1}{2}+\frac{d}{2 q}, \infty}$ and that $H^{ \pm 2, q}\left(\mathbb{R}^{d}\right)^{d}=X_{ \pm 1}(-\Delta)$ for $X=L^{q}\left(\mathbb{R}^{d}\right)^{d}$. This is due to

$$
\left\|u_{0}\right\|_{B_{q, \infty}^{-1+d / q}\left(\mathbb{R}^{d}\right)^{d}} \approx\left\|t \mapsto t^{\frac{1}{2}-\frac{d}{2 q}} e^{-t} e^{t \Delta} u_{0}\right\|_{L^{\infty}\left(\mathbb{R}_{+} ; L^{q}\left(\mathbb{R}^{d}\right)^{d}\right)} .
$$

By Theorem 4.1 the heat semigroup $\left(e^{t \Delta}\right)_{t \geq 0}$ on $B_{q, \infty}^{-1+d / q}\left(\mathbb{R}^{d}\right)^{d}$ is thus bi-continuous with respect to $\tau$ and the same holds on the invariant closed subspace $B_{q, \infty, \sigma}^{-1+d / q}\left(\mathbb{R}^{d}\right)$.

For $\alpha>0$ and a Banach space $Z$ denote by $L_{\alpha}^{\infty}(0, a ; Z)$ the space of measurable functions $f:(0, a) \rightarrow Z$ such that $\|f\|_{L_{\alpha}^{\infty}(Z)}:=$ ess sup $\left\|t^{\alpha} f(t)\right\|_{Z}<\infty$. The fixed point argument is carried out in $L_{\alpha}^{\infty}\left(0, a ; L^{q}\left(\mathbb{R}^{d}\right)^{d}\right)$ for $\alpha=\frac{1}{2}-\frac{d}{2 q}$. For $v \in L_{\alpha}^{\infty}\left(L^{q}\left(\mathbb{R}^{d}\right)^{d}\right)$ we have $\operatorname{div}(v \otimes v) \in L_{2 \alpha}^{\infty}\left(H^{-1, q / 2}\left(\mathbb{R}^{d}\right)^{d}\right)$. Since $e^{t \Delta}$ : $H^{-1, q / 2}\left(\mathbb{R}^{d}\right) \rightarrow L^{q}\left(\mathbb{R}^{d}\right)$ has norm $\leq C t^{-\frac{1}{2}-\frac{d}{2 q}}$ on a bounded interval we can check directly that convolution with the heat semigroup is bounded $L_{2 \alpha}^{\infty}\left(0, a ; H^{-1, q / 2}\left(\mathbb{R}^{d}\right)^{d}\right) \rightarrow$ $L_{\alpha}^{\infty}\left(0, a ; L^{q}\left(\mathbb{R}^{d}\right)^{d}\right)$. So, if $u_{0}$ has small norm, one can make the fixed point argument work in $L_{\alpha}^{\infty}\left(0, a ; L^{q}\left(\mathbb{R}^{d}\right)^{d}\right)$ and even in $L_{\alpha}^{\infty}\left(0, a ; L^{q}\right) \cap C\left((0, a], L^{q}\right)$ by analyticity of the semigroup. Another direct calculation shows that convolution with the heat semigroup is bounded $L_{2 \alpha}^{\infty}\left(0, a ; H^{-1, q / 2}\left(\mathbb{R}^{d}\right)^{d}\right) \rightarrow C_{b}\left((0, a], B_{q, \infty}^{-1+d / q}\left(\mathbb{R}^{d}\right)^{d}\right)$.

By standard means one can now show (see., e.g., [7]) that (17) has a unique solution in $C_{b}\left((0, a] ; B_{q, \infty}^{-1+d / q}\left(\mathbb{R}^{d}\right)^{d}\right) \cap L_{\alpha}^{\infty}\left(0, a ; L^{q}\left(\mathbb{R}^{d}\right)\right)$. The heat semigroup has a densely defined generator in $H^{-1, q / 2}\left(\mathbb{R}^{d}\right)^{d}$, and this can be used to show that, denoting the convolution term on the right-hand side of (17) by $w$, we have $w(t) \rightarrow$ 0 in $B^{-1+d / q}\left(\mathbb{R}^{d}\right)^{d}$ as $t \rightarrow 0$. We conclude that (17) has a unique solution in $C_{\tau}\left([0, a], B_{q, \infty, \sigma}^{-1+d / q}\left(\mathbb{R}^{d}\right)\right) \cap L_{1 / 2-d /(2 q)}^{\infty}\left(0, a ; L_{\sigma}^{q}\left(\mathbb{R}^{d}\right)\right)$ if the norm of $u_{0}$ is sufficiently small. 


\section{Acknowledgements}

The author wants to thank the unknown referee for careful reading of the manuscript and several suggestions that helped to improve the presentation, in particular for mentioning references $[2,6,12]$.

\section{Funding Open Access funding enabled and organized by Projekt DEAL.}

Open Access. This article is licensed under a Creative Commons Attribution 4.0 International License, which permits use, sharing, adaptation, distribution and reproduction in any medium or format, as long as you give appropriate credit to the original author(s) and the source, provide a link to the Creative Commons licence, and indicate if changes were made. The images or other third party material in this article are included in the article's Creative Commons licence, unless indicated otherwise in a credit line to the material. If material is not included in the article's Creative Commons licence and your intended use is not permitted by statutory regulation or exceeds the permitted use, you will need to obtain permission directly from the copyright holder. To view a copy of this licence, visit http://creativecommons.org/licenses/ by/4.0/.

Publisher's Note Springer Nature remains neutral with regard to jurisdictional claims in published maps and institutional affiliations.

\section{REFERENCES}

[1] H. Amann, On the strong solvability of the Navier-Stokes equations, J. Math. Fluid Mech. 2, 16-98 (2000).

[2] C. Budde, B. Farkas, Intermediate and extrapolated spaces for bi-continuous operator semigroups. J. Evol. Equ. 19 (2), 321-359 (2019).

[3] M. Cannone, Ondelettes, paraproduits et Navier-Stokes, Diderot Editeur, Paris, 1995.

[4] M.G. Cowling, I. Doust, A. McIntosh, A. Yagi, Banach space operators with a bounded $H^{\infty}$ functional calculus, J. Aust. Math. Soc. A 60 (1), 51-89 (1996).

[5] G. Dore, $H^{\infty}$-functional calculus in real interpolation spaces, Studia Math. 137 (2), 161-167 (1999).

[6] B. Farkas, Perturbations of bi-continuous semigroups. Studia Math. 161 (2), 147-161 (2004).

[7] B.H. Haak, P.C. Kunstmann, On Kato's method for Navier Stokes equations, J. Math. Fluid Mech. 11, 492-535 (2009).

[8] B.H. Haak, M. Haase, P.C. Kunstmann, Perturbation, interpolation, and maximal regularity, Adv. Differential Equations 11, 201-240 (2006).

[9] M. Haase, The Functional Calculus for Sectorial Operators, Operator Theory: Advances and Applications, vol. 169, Birkhäuser, Basel (2006).

[10] M. Hieber, Laplace transforms and $\alpha$-times integrated semigroups, Forum Math. 3, 595-612 (1991).

[11] M. Hieber, J. Prüss, Functional calculi for linear operators in vector-valued $L^{p}$-spaces via the transference principle, Advances in Differential Equations 3 (6), 847-872 (1998).

[12] P. Jara, Rational approximation schemes for bi-continuous semigroups. J. Math. Anal. Appl. 344 (2), 956-968 (2008).

[13] F. Kühnemund, A Hille-Yosida theorem for bi-continuous semigroups, Semigroup Forum 67 (2), 205-255 (2003).

[14] P.C. Kunstmann, Modulation type spaces for generators of polynomially bounded groups and Schrödinger equations, Semigroup Forum 98 (3), 645-668 (2019).

[15] P.C. Kunstmann, Around maximal regularity for Navier-Stokes equations, submitted.

[16] H. Triebel, Interpolation Theory, Function Spaces, Differential Operators, North-Holland Mathematical Library, vol. 18. North-Holland, Amsterdam (1978). 


\author{
Peer Christian Kunstmann \\ Institute for Analysis \\ Karlsruhe Institute of Technologie (KIT) \\ Englerstr. 2 \\ 76128 Karlsruhe \\ Germany \\ E-mail: peer.kunstmann@kit.edu
}

Accepted: 11 November 2020 